\title{
A note on a modification of Gajda's equation
}

\author{
ŻYWILLA FECHNER
}

\begin{abstract}
In the present paper we deal with an integral generalization of a d'Alembert-type equation for unknown mappings defined on a locally compact Abelian group and taking their values in the field of complex numbers.
\end{abstract}

Mathematics Subject Classification (2000). 42Axx, 45Xxx.

Keywords. Cosine function, integral-functional equation, d'Alembert equation, Wilson's equation, Gajda's equation.

\section{Introduction}

Let $(G,+)$ be a locally compact Abelian group, $(\Gamma, \cdot)$ be the group of all continuous characters of $G$ and $m$ be a fixed Haar measure on $G$. Further, let $M(G)$ denote the space of all regular bounded complex-valued Borel measures on $G$. Let

$$
L^{1}(G)=\left\{f: G \rightarrow \mathbb{C}: f \text { is } m-\text { measurable and } \int_{G}|f| d m<\infty\right\}
$$

be equipped with the norm $\|f\|_{1}=\int_{G}|f| d m$. For $\mu \in M(G)$ and $y \in G$ we put:

$$
\mu^{-}(A):=\mu(-A), \quad \mu_{y}(A):=\mu(A+y)
$$

for $A \in \mathcal{B}(G)$, where $\mathcal{B}(G)$ denotes the space of all Borel subsets of $G$. By $L^{\infty}(G)$ we mean the space of all $m$-measurable functions $f: G \rightarrow \mathbb{C}$ which are bounded $m$-locally almost everywhere ( $m$-l.a.e. $)$, i.e. outside a set $A_{f} \subset G$ such that for all compact sets $K \subset G$ it is $m\left(A_{f} \cap K\right)=0$. The space $L^{\infty}(G)$ is equipped with the following norm:

$$
\|f\|_{\infty}:=\inf \{a \geq 0:|f| \leq a \quad m \text { - l.a.e. }\} .
$$


Next, for a Borel function $f \in L^{\infty}(G)$ and $\mu \in M(G)$ we define the convolution of $f$ and $\mu$ by the formula:

$$
(f * \mu)(x)=\int_{G} f(x-t) d \mu(t), \quad x \in G .
$$

For further discussion of measurability see Remark 8 below. It is well known that if $f \in L^{\infty}(G)$ is a Borel function and $\mu \in M(G)$ then $f * \mu \in L^{\infty}(G)$.

Throughout the paper we will use the following notation: for any function $F: G \rightarrow \mathbb{C}$ we define the mapping $\check{F}: G \rightarrow \mathbb{C}$ by

$$
\check{F}(x):=F(-x), \quad x \in G .
$$

All the terminology concerning the field of harmonic analysis is in accordance with the monographs of Hewitt and Ross [4,5].

Now we introduce some functional equations concerning trigonometric functions. The classical d'Alembert's functional equation is of the form

$$
f(x+y)+f(x-y)=2 f(x) f(y), \quad x, y \in G .
$$

It is also known as the cosine functional equation since $f=\cos$ satisfies (2) in the scalar case. Equation (2) has been investigated by many authors in different settings. There are several possible generalizations of d'Alembert's equation known. One of them is the classical Wilson's equation for the pair $(g, f)$ :

$$
g(x+y)+g(x-y)=2 g(x) f(y), \quad x, y \in G .
$$

For details and further references concerning Eqs. (2) and (3) see e.g. the monograph of Aczél and Dhombres [1] and the monograph of Székelyhidi [7].

Another possible extension of d'Alembert's functional equation is the following equation:

$$
g(x+y)+g(x-y)=2 f(x) g(y), \quad x, y \in G .
$$

A generalization of (2) in a different direction is due to Gajda [3]. He studied the following integral equation:

$$
\left(f * \mu_{y}\right)(x)+\left(f *\left(\mu_{y}\right)^{-}\right)(x)=f(x) f(y), \quad x, y \in G,
$$

for the function from $f \in L^{\infty}(G)$ which does not vanish $m$-l.a.e. and for the measure $\mu \in M(G)$. The following Wilson's-type generalization of Gajda's equation:

$$
\left(g * \mu_{y}\right)(x)+\left(g *\left(\mu_{y}\right)^{-}\right)(x)=g(x) f(y), \quad x, y \in G,
$$

was considered in [2].

In the present paper we are going to deal with the following generalization of Gajda's equation:

$$
\left(g * \mu_{y}\right)(x)+\left(g *\left(\mu_{y}\right)^{-}\right)(x)=f(x) g(y), \quad x, y \in G .
$$

Note that this equation is a joint generalization of (4) and (5). Now we quote the main result from [3]: 
Theorem 1. (Z. Gajda [3]) Let $\mu \in M(G)$ be arbitrarily fixed. Then a function $f \in L^{\infty}(G)$ which does not vanish $m$-l.a.e. satisfies Eq. (5) if and only if there exists a character $\gamma \in \Gamma$ such that

$$
f(y)=\left(\gamma * \mu_{y}\right)(0)+\left(\gamma *\left(\mu_{y}\right)^{-}\right)(0)=\int_{G} \gamma(y-s)+\gamma(s-y) d \mu(s)
$$

for all $y \in G$.

It will be convenient to recall the general solution of (6) obtained in [2].

Theorem 2. Let $\mu \in M(G)$ and let $f, g \in L^{\infty}(G)$ be Borel functions which do not vanish $m$-l.a.e. If the pair $(g, f)$ satisfies Eq. (6), then there exist a character $\gamma \in \Gamma$ and constants $C_{1}, C_{2} \in \mathbb{C}$ such that $f$ is of the form (8) and

$$
g(x)=C_{1} \gamma(x)-C_{2} \check{\gamma}(x), \quad x \in G .
$$

Conversely, if $\gamma \in \Gamma$ is a character, $C_{1}, C_{2} \in \mathbb{C}$ are constants and $f$ is given by (8) and $g$ by (9), then the pair $(g, f)$ fulfills (6).

We will make use of the general form of bounded solutions of Wilson's functional equation. This result is based on the form of solutions of the classical Wilson's functional equation for functions defined on an Abelian group given by Székelyhidi in [7] and on some results obtained by Stetkær in [6].

Lemma 3. Let $f, g \in L^{\infty}(G)$ be functions which do not vanish m-l.a.e. The pair $(g, f)$ satisfies Wilson's functional equation if and only if either:

(i) there exist a character $\chi_{1} \in \Gamma$ and a constant $\alpha \in \mathbb{C}$ such that $\chi_{1}^{2}=1$ and

$$
f(x)=\chi_{1}(x), \quad g(x)=\alpha \chi_{1}(x), \quad x \in G,
$$

or

(ii) there exist a character $\chi \in \Gamma$ and constants $K, L \in \mathbb{C}$ such that $\chi^{2} \neq 1$ and

$$
f(x)=\frac{\chi(x)+\check{\chi}(x)}{2}, \quad g(x)=K \chi(x)+L \check{\chi}(x), \quad x \in G .
$$

The proof of Lemma 3 can be found in [2].

\section{Main result}

We begin with a description of solutions of (4).

Theorem 4. Let $(G,+)$ be an Abelian group and $g, f: G \rightarrow \mathbb{C}$. If the pair $(g, f)$ is a solution of (4), then either:

(i) $g=0$ and $f$ is arbitrary;

or

(ii) $g \neq 0, g=g(0) f$ and $f$ satisfies the d'Alembert functional equation. 
Proof. Interchanging the roles of $x$ and $y$ in (4) leads to

$$
g(x+y)+g(y-x)=2 f(y) g(x), \quad x, y \in G .
$$

Now putting $y=0$ in (12) we arrive at

$$
g(x)+g(-x)=2 f(0) g(x), \quad x \in G .
$$

Let us consider two cases:

Case 1. $f(0)=0$.

Then $g$ is odd. Adding (4) and (12) side by side we get

$$
g(x+y)=g(x) f(y)+f(x) g(y), \quad x, y \in G .
$$

Consequently, for $y=0$ we have $g(x)=f(x) g(0)$ for all $x \in G$. In particular, $g(0)=f(0) g(0)=0$ and thus $g=0$. Therefore, $f$ can be arbitrary.

Case 2. $f(0) \neq 0$.

By (13) we have

$$
g(x)=\frac{1}{f(0)} \frac{g(x)+g(-x)}{2}, \quad x \in G
$$

Thus $g$ is even. We may assume that $g \neq 0$. Applying (13) and by the evenness of $g$ we get $2 g(x)=2 f(0) g(x)$ for all $x \in G$. Thus $f(0)=1$. Comparing (4) with (12) we deduce the equality

$$
f(x) g(y)=f(y) g(x), \quad x, y \in G \text {. }
$$

Put $x=0$ to obtain $g=g(0) f$. On joining this with (4) we get that $f$ satisfies the d'Alembert equation.

Let us rewrite Eq. (7) in the following way:

$$
\int_{G}[g(x+y-s)+g(x-y+s)] d \mu(s)=f(x) g(y), \quad x, y \in G .
$$

Now we prove the key lemma:

Lemma 5. Let $\mu \in M(G)$ and let $g \in L^{\infty}(G)$ be a Borel function and let $f: G \rightarrow \mathbb{C}$ be arbitrary. If the pair $(g, f)$ satisfies equation $(7)$ then

$$
g(y) f(x)=g(x) f(y), \quad x, y \in G .
$$

Moreover, if $g \neq 0$ then $f \in L^{\infty}(G)$ and $f$ is Borel measurable. 
Proof. We may assume that $f \neq 0$. Otherwise the statement is trivial. Take $x_{0} \in G$ such that $f\left(x_{0}\right) \neq 0$. Applying Eq. (14) repeatedly we get

$$
\begin{aligned}
f\left(x_{0}\right) f(x) g(y)= & f\left(x_{0}\right) \int_{G}[g(x+y-s)+g(x-y+s)] d \mu(s) \\
= & \int_{G} f\left(x_{0}\right) g(x+y-s) d \mu(s)+\int_{G} f\left(x_{0}\right) g(x-y+s) d \mu(s) \\
= & \int_{G} \int_{G}\left[g\left(x_{0}+x+y-s-t\right)+g\left(x_{0}-x-y+s+t\right)\right] d \mu(t) d \mu(s) \\
& +\int_{G} \int_{G}\left[g\left(x_{0}+x-y+s-t\right)+g\left(x_{0}-x+y-s+t\right)\right] d \mu(t) d \mu(s) \\
= & \int_{G} \int_{G}\left[g\left(x_{0}+y+x-s-t\right)+g\left(x_{0}-y-x+s+t\right)\right] d \mu(t) d \mu(s) \\
& +\int_{G} \int_{G}\left[g\left(x_{0}+y-x+s-t\right)+g\left(x_{0}-y+x-s+t\right)\right] d \mu(t) d \mu(s)
\end{aligned}
$$

The second term in the last line of the above sum has been obtained by the Fubini theorem and simultaneously the names of variables $s$ and $t$ have been interchanged. We see that the right-hand-side is symmetric with respect to $x$ and $y$, thus

$$
f\left(x_{0}\right) f(x) g(y)=f\left(x_{0}\right) f(y) g(x), \quad x, y \in G .
$$

Since $f\left(x_{0}\right) \neq 0$ then we get

$$
g(y) f(x)=g(x) f(y), \quad x, y \in G .
$$

If we additionally assume that $g \neq 0$ then there exists an $x_{0} \in G$ such that $g\left(x_{0}\right) \neq 0$. Thus applying the above equation for $y=x_{0}$ we arrive at

$$
f(x)=g\left(x_{0}\right)^{-1} f\left(x_{0}\right) g(x), \quad x \in G .
$$

Thus taking a constant $C:=g\left(x_{0}\right)^{-1} f\left(x_{0}\right)$ we obtain

$$
f(x)=C g(x), \quad x \in G
$$

and therefore from the $m$-l.a.e. boundedness of $g$ we get the $m$-l.a.e. boundedness of $f$. Similarly, if $g$ is measurable then $f$ is also measurable with respect to the same $\sigma$-field.

Now we are ready to state and prove our main result.

Theorem 6. Let $\mu \in M(G)$ and let $g \in L^{\infty}(G)$ be a Borel function which does not vanish $m$-l.a.e. and $f: G \rightarrow \mathbb{C}$ be $m$-measurable. Assume that $f \neq 0$. If the pair $(g, f)$ satisfies (14) then $f$ is bounded and there exist a character $\gamma \in \Gamma$ and a constant $C \in \mathbb{C}$ such that $f$ is given by (8) and $g=C f$.

Conversely, if $f$ is given by (8) and there exists a constant $C$ such that $g=C f$, then the pair $(g, f)$ satisfies (14). 
Proof. By Lemma 5 we infer that if the pair $(g, f)$ satisfies $(14)$ then there exists a constant $C \in \mathbb{C}$ such that $g=C f$. If $C=0$, then $g=0$, which is impossible since $g$ does not vanish $m$-l.a.e. Therefore $C \neq 0$. Equation (14) is now of the form

$$
C \int_{G}[f(x+y-s)+f(x-y+s)] d \mu(s)=C f(x) f(y), \quad x, y \in G .
$$

The constant $C \neq 0$, thus we get Eq. (5). By Gajda's Theorem 1 we deduce that there exists a character $\gamma \in \Gamma$ such that $f$ is given by (8). Therefore we obtain the desired form of solutions. The converse part of the statement is a straightforward calculation and therefore is omitted.

Remark 7. In Theorem 4 we proved that in the case of solutions of equation (4) function $g$ is proportional to function $f$, which is additionally a solution of the d'Alembert functional equation (2). In Theorem 6 we obtained similar results for the solution of Eq. (7): map $g$ from the solution of Eq. (7) is proportional to a map $f$ which additionally is a solution of Gajda's equation (5). The constant $C$ depends on the measure $\mu$ which appears in the equation. The same effect holds for the corresponding Wilson's-type generalization of Gajda's equation, which was described in Lemma 2 , where constants $C_{1}$ and $C_{2}$ also depend on the choice of a measure $\mu$.

Remark 8. For a Borel function $f \in L^{\infty}(G)$ and $\mu \in M(G)$ we have already defined the convolution by the formula (1), thus we need a Borel measurability of $f$. If $f \in L^{\infty}(G)$ is also integrable with respect to $m$ then by Corollary (11.41) from [4] we need not assume that $f$ is a Borel function. Similarly, if $G$ is a $\sigma$-compact space then by Lemma (20.7) from [4] we have that every $m$-measurable function $f$ is equal $m$-a.e. to some Borel function. Thus in Lemma 5 and in Theorems 2 and 6 the assumption of Borel measurability may be omitted in the above cases.

\section{Acknowledgements}

I am grateful for all the reviewers' suggestions. In particular, the proof of Theorem 6 was significantly shortened thanks to the referee's remarks.

Open Access. This article is distributed under the terms of the Creative Commons Attribution Noncommercial License which permits any noncommercial use, distribution, and reproduction in any medium, provided the original author(s) and source are credited.

\section{References}

[1] Aczél, J., Dhombres, J.: Functional equations in several variables, Encyclopedia Math. Appl. 31, Cambridge Univ. Press, Cambridge (1989) 
[2] Fechner, Ż.: A generalization of Gajda's equation. J. Math. Anal. Appl. 354, 584-593 (2009)

[3] Gajda, Z.: A generalization of d'Alembert's functional equation. Funkcialaj Ekvacioj 33, 69-77 (1990)

[4] Hewitt, E., Ross, K.A.: Abstract harmonic analysis. Vol. I: Structure of topological groups. Integration theory, group representation, Die Grundlehren der mathematischen Wissenschaften, Bd. 115 Academic Press, Inc., Publishers, New York; Springer, Berlin (1963)

[5] Hewitt, E., Ross, K.A.: Abstract harmonic analysis, Vol II: Structure and analysis for compact groups, Analysis on locally compact Abelian groups. Die Grundlehren der mathematischen Wissenschaften Band 152 Springer-Verlag, Berlin (1970)

[6] Stetkær, H.: D'Alembert's functional equations on metabelian groups. Aequationes Math. 59, 306-320 (2000)

[7] Székelyhidi, L.: Convolution type functional equation on topological Abelian groups. World Scientific, Singapore (1991)

Żywilla Fechner

Institute of Mathematics

University of Silesia

Bankowa 14, 40-007 Katowice

Poland

e-mail: zfechner@gmail.com

Received: October 17, 2010

Revised: March 31, 2011 